\title{
Graph Realizations Constrained by Connected Local Dimensions and Connected Local Bases
}

\author{
VARANOOT KHEMMANI $^{1}$, WITSARUT PHO-ON ${ }^{2}$, SUPACHOKE ISARIYAPALAKUL ${ }^{3}$ \\ ${ }^{1,2,3}$ Department of Mathematics, Srinakharinwirot University, \\ Sukhumvit 23, Bangkok 10110, THAILAND
}

\begin{abstract}
For an ordered set $W=\left\{w_{1}, w_{2}, \ldots, w_{k}\right\}$ of $k$ distinct vertices in a connected graph $G$, the representation of a vertex $v$ of $G$ with respect to $W$ is the $k$-vector $r(v \mid W)=\left(d\left(v, w_{1}\right), d\left(v, w_{2}\right), \ldots, d\left(v, w_{k}\right)\right)$, where $d\left(v, w_{i}\right)$ is the distance from $v$ to $w_{i}$ for $1 \leq i \leq k$. The set $W$ is called a connected local resolving set of $G$ if the representations of every two adjacent vertices of $G$ with respect to $W$ are distinct and the subgraph $\langle W\rangle$ induced by $W$ is connected. A connected local resolving set of $G$ of minimum cardinality is a connected local basis of $G$. The connected local dimension $\operatorname{cld}(G)$ of $G$ is the cardinality of a connected local basis of $G$. In this paper, the connected local dimensions of some well-known graphs are determined. We study the relationship between connected local bases and local bases in a connected graph, and also present some realization results.
\end{abstract}

Key-Words: representation, connected local resolving set, connected local basis, connected local dimension.

Received: March 5, 2021. Revised: October 6, 2021. Accepted: December 19. Published: January 2, 2022.

\section{Introduction}

For an ordered set $W=\left\{w_{1}, w_{2}, \ldots, w_{k}\right\}$ of $k$ distinct vertices of a connected graph $G$, the representation of a vertex $v$ of $G$ with respect to $W$ is the $k$-vector

$$
r(v \mid W)=\left(d\left(v, w_{1}\right), d\left(v, w_{2}\right), \ldots, d\left(v, w_{k}\right)\right),
$$

where $d\left(v, w_{i}\right)$ is the distance between $v$ and $w_{i}$ for each integer $i$ with $1 \leq i \leq k$. If representations of any pairs of vertices $u$ and $v$ with respect to $W$ are distinct, then $W$ is called a resolving set of $G$. A resolving set of minimum cardinality is a minimum resolving set or a basis of $G$. The cardinality of basis of $G$ is the dimension of $G$, which is denoted by $\operatorname{dim}(G)$. To illustrate this concept, consider the graph $G$ of Fig. 1. We consider the representations of vertices of $G$ with

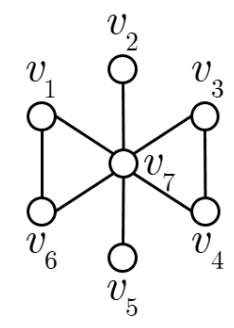

Figure 1: A connected graph $G$

respect to the ordered set $W_{1}=\left\{v_{1}, v_{3}\right\}$. Therefore, their representations with respect to $W_{1}$ are

$$
\begin{array}{ll}
r\left(v_{1} \mid W_{1}\right)=(0,2), & r\left(v_{2} \mid W_{1}\right)=(2,2), \\
r\left(v_{3} \mid W_{1}\right)=(2,0), & r\left(v_{4} \mid W_{1}\right)=(2,1), \\
r\left(v_{5} \mid W_{1}\right)=(2,2), & r\left(v_{6} \mid W_{1}\right)=(1,2), \\
r\left(v_{7} \mid W_{1}\right)=(1,1) . &
\end{array}
$$

Since $r\left(v_{2} \mid W_{1}\right)=(2,2)=r\left(v_{5} \mid W_{1}\right)$, it follows that $W_{1}$ is not a resolving set of $G$. By considering the ordered set $W_{2}=\left\{v_{1}, v_{2}, v_{3}\right\}$, the representations of vertices of $G$ with respect to $W_{2}$ are

$$
\begin{array}{ll}
r\left(v_{1} \mid W_{2}\right)=(0,2,2), & r\left(v_{2} \mid W_{2}\right)=(2,0,2), \\
r\left(v_{3} \mid W_{2}\right)=(2,2,0), & r\left(v_{4} \mid W_{2}\right)=(2,2,1), \\
r\left(v_{5} \mid W_{2}\right)=(2,2,2), & r\left(v_{6} \mid W_{2}\right)=(1,2,2), \\
r\left(v_{7} \mid W_{2}\right)=(1,1,1) . &
\end{array}
$$

Since these representations are distinct, it follows that $W_{2}$ is a resolving set of $G$. In fact, $W_{2}$ is a basis of $G$ and so $\operatorname{dim}(G)=3$.

The concept of resolving sets was introduced by Slater in [13] and [14]. He used a locating set for what we have called a resolving set and referred to the cardinality of a basis of a connected graph as its location number. He described the usefulness of this idea when working with U.S. sonar and coast guard LORAN (long range aids to navigation) stations. Following Slater and others [4], [5] and [6], we can think of a resolving set as the set $W$ of vertices in a connected graph $G$ so that each vertex in $G$ is uniquely determined by its distances to the vertices of $W$. Independently, Harary and Melter [3] discovered this concept as well but used the term metric dimension rather than location number. This concept was rediscovered 
by Johnson [8] of the Pharmacia Company while attempting to develop a capability of large datasets of chemical graphs. In [1], Chartrand and others used the term resolving set for locating set and used metric dimension for location number. Wang, Miao and Liu [15] characterized connected graphs of order $n$ with dimension $n-3$ by using metric matrix. An application of resolving set was presented in [11]. Resolving sets in graphs have been studied further in [7], [9] and [10].

Let $W$ be an ordered set of vertices of a connected graph $G$. For every pair of adjacent vertices $u$ and $v$ in $G$, if the representations of $u$ and $v$ with respect to $W$ are distinct, then $W$ is called a local resolving set of $G$. A local resolving set of $G$ having minimum cardinality is a minimum local resolving set or a local basis of $G$ and this cardinality is the local dimension of $G$, which is denoted by $\operatorname{ld}(G)$. A subgraph $H$ of a graph $G$ is called an induced subgraph of $G$ if whenever $u$ and $v$ are vertices of $H$ and $u v$ is an edge of $G$, then $u v$ is an edge of $H$ as well. If $S$ is a nonempty set of vertices of $G$, then the subgraph of $G$ induced by $S$ is the induced subgraph with vertex set $S$. This induced subgraph is denoted by $\langle S\rangle$. A resolving set $W$ of a connected graph $G$ is called a connected resolving set of $G$ if the induced subgraph $\langle W\rangle$ is connected. The minimum cardinality of a connected resolving set of $G$ is the connected dimension of $G$, which is denoted by $\operatorname{cd}(G)$, and a resolving set of $G$ having this cardinality is called a minimum connected resolving set of a connected basis of $G$. To illustrate these concepts, consider the graph $G$ of Fig. 1. Recall that $W_{1}=\left\{v_{1}, v_{3}\right\}$ is not a resolving set of $G$. However, since the representations of any two adjacent vertices of $G$ with respect to $W_{1}$ are distinct, it follows that $W_{1}$ is a local resolving set of $G$. Clearly, there is no a local resolving set of $G$ consisting of one vertex. Therefore, $W_{1}$ is a local basis of $G$ and so $\operatorname{ld}(G)=2$. For an ordered set $W_{2}=\left\{v_{1}, v_{2}, v_{3}\right\}$, we know that $W_{2}$ is a resolving set of $G$. Since $\left\langle W_{2}\right\rangle$ is not connected, it follows that $W_{2}$ is not a connected resolving set of $G$. It is routine to verify that $W_{3}=\left\{v_{1}, v_{2}, v_{3}, v_{7}\right\}$ is a connected resolving set of $G$. Indeed, $W_{3}$ is a connected basis of $G$, that is, $\operatorname{cd}(G)=4$.

The concept of local resolving sets was introduced by Okamoto and others in [2]. They characterized all nontrivial connected graphs of order $n$ having the local dimension $1, n-2$ or $n-1$. The idea of connected resolving sets has appeared in [12] and used the connected resolving number $\operatorname{cr}(G)$ of a connected graph $G$ for what we have called the connected dimension of $G$. The local dimension and the connected dimension of some well-known graphs have been determined in [2] and [12], respectively. We state these results in the next three theorems.
Theorem 1.1 ([2]). Let $G$ be a nontrivial connected graph of order $n$. Then

(i) $\operatorname{ld}(G)=n-1$ if and only if $G=K_{n}$ and

(ii) $\operatorname{ld}(G)=1$ if and only if $G$ is bipartite.

Theorem 1.2 ([12]). Let $G$ be a nontrivial connected graph of order $n$. Then

(i) $\operatorname{cd}(G)=1$ if and only if $G=P_{n}$ and

(ii) if $G=C_{n}$, then $\operatorname{cd}(G)=2$.

Theorem 1.3 ([12]). Let $G$ be a connected graph of order $n \geq 3$. Then $\operatorname{cd}(G)=n-1$ if and only if $G=K_{n}$ or $G=K_{1, n-1}$.

In this paper, we study a local resolving set $W$ of a connected graph $G$ such that the induced subgraph $\langle W\rangle$ is connected in $G$. In order to do this, let us introduce some definitions and notation. Let $W$ be an ordered set of vertices of a connected graph $G$. Then $W$ is called a connected local resolving set of $G$ if $W$ is a local resolving set of $G$ such that the induced subgraph $\langle W\rangle$ of $G$ is connected. A connected local resolving set of $G$ having minimum cardinality is a minimum connected local resolving set or a connected local basis of $G$ and this cardinality is the connected local dimension of $G$, which is denoted by $\operatorname{cld}(G)$. To illustrate this concept, consider the graph $G$ of Fig. 1 . We know that $W_{1}=\left\{v_{1}, v_{3}\right\}$ is a local resolving set of $G$. However, since the induced subgraph $\left\langle W_{1}\right\rangle$ of $G$ is not connected, it follows that $W_{1}$ is not a connected local resolving set of $G$. Then consider the ordered set $W^{\prime}=\left\{v_{1}, v_{3}, v_{7}\right\}$. The representations of vertices of $G$ with respect to $W^{\prime}$ are

$$
\begin{array}{ll}
r\left(v_{1} \mid W^{\prime}\right)=(0,2,1), & r\left(v_{2} \mid W^{\prime}\right)=(2,2,1), \\
r\left(v_{3} \mid W^{\prime}\right)=(2,0,1), & r\left(v_{4} \mid W^{\prime}\right)=(2,1,1), \\
r\left(v_{5} \mid W^{\prime}\right)=(2,2,1), & r\left(v_{6} \mid W^{\prime}\right)=(1,2,1), \\
r\left(v_{7} \mid W^{\prime}\right)=(1,1,0) . &
\end{array}
$$

Since the representations of any two adjacent vertices of $G$ with respect to $W^{\prime}$ are distinct, if follows that $W^{\prime}$ is a local resolving set of $G$. Moreover, the induced subgraph $\left\langle W^{\prime}\right\rangle$ is connected and so $W^{\prime}$ is a connected local resolving set of $G$. By a case-by-case analysis, it can be shown that every connected local resolving set of $G$ must contain at least two vertices, that is, one of $\left\{v_{1}, v_{6}\right\}$ and one of $\left\{v_{3}, v_{4}\right\}$. Thus, there is no connected resolving set of $G$ having cardinality 2 and so $W^{\prime}$ is a connected local basis of $G$. Hence, $\operatorname{cld}(G)=3$.

Observe that every connected local resolving set of a connected graph $G$ is also a local resolving set of $G$ but every local resolving set of $G$ may or may not be a connected local resolving set of $G$. This implies that

$$
1 \leq \operatorname{ld}(G) \leq \operatorname{cld}(G) \leq n-1 .
$$


If $W$ is a connected local resolving set of $G$, then $\langle W\rangle$ is connected. However, since the representations of any two vertices of $G$ with respect to $W$ need not be distinct, it follows that $W$ is not necessarily a connected resolving set of $G$. In fact, every connected resolving set of $G$ is a connected local resolving set of $G$, that is,

$$
1 \leq \operatorname{cld}(G) \leq \operatorname{cd}(G) \leq n-1 .
$$

From Eq.(1.1) and Eq.(1.2), we obtain that

$$
1 \leq \operatorname{ld}(G) \leq \operatorname{cld}(G) \leq \operatorname{cd}(G) \leq n-1 .
$$

For every ordered set $W=\left\{w_{1}, w_{2}, \ldots, w_{k}\right\}$ of vertices of a connected graph $G$, recall that the only vertex of $G$ whose representation with respect to $W$ contains 0 in its $i^{\text {th }}$ coordinate is $w_{i}$, that it, the vertices of $W$ necessarily have distinct representations with respect to $W$. On the other hand, the representations of vertices of $G$ that do not belong to $W$ have elements, all of which are positive. Indeed, to determine whether an ordered set $W$ is a connected local resolving set of $G$, we only need to show that any two adjacent vertices in $V(G)-W$ have distinct representations with respect to $W$ and $\langle W\rangle$ is connected.

\section{The connected local dimensions of some well-known graphs}

In this section, we determined the connected local dimensions of some well-known graphs.

Theorem 2.1. Let $G$ be a connected graph of order $n \geq 2$. Then

(i) $\operatorname{cld}(G)=1$ if and only if $G$ is a bipartite graph,

(ii) $\operatorname{cld}(G)=n-1$ if and only if $G=K_{n}$, a complete graph of order $n$.

Proof. (i) Assume that $\operatorname{cld}(G)=1$. Then $\operatorname{ld}(G)=1$ by Eq.(1.3). Therefore, $G$ is bipartite by Theorem 1.1 (ii). For converse, suppose that $G$ is bipartite. By Theorem 1.1 (ii), $\operatorname{ld}(G)=1$ and so there is a 1-element local basis $W$ of $G$. Indeed, $W$ is also connected local basis of $G$, that is, $\operatorname{cld}(G)=1$.

(ii) Suppose that $\operatorname{cld}(G)=n-1$. Eq.(1.2) implies that $\operatorname{cd}(G)=n-1$. Thus, by Theorem 1.3, $G$ is complete or star. If $G$ is a star that is not complete, then $G$ is bipartite of order at least 3. By (i), $\operatorname{cld}(G)=$ 1 , a contradiction. Hence, $G$ is complete. On the other hand, if $G=K_{n}$, then by Theorem 1.1, $\operatorname{ld}(G)=$ $n-1$, and so $\operatorname{cld}(G)=n-1$ by Eq.(1.3).

Since every bipartite graph contains no odd cycle, it follows that the connected local dimension of an even cycle is 1 . In fact, the connected local dimension of an odd cycle is 2 , as we present next.
Theorem 2.2. For an integer $n \geq 3$, the connected local dimension of a cycle $C_{n}$ is

$$
\operatorname{cld}\left(C_{n}\right)=\left\{\begin{array}{l}
1 \text { if } n \text { is even }, \\
2 \quad \text { if } n \text { is odd } .
\end{array}\right.
$$

Proof. If $n$ is even, then $C_{n}$ is bipartite. By Theorem 2.1 (i), $\operatorname{cld}(G)=1$. We may assume that $n$ is odd. Let $C_{n}=\left(v_{1}, v_{2}, \ldots, v_{n}, v_{1}\right)$ and let $W=$ $\left\{v_{1}, v_{2}\right\}$. Consider the representations of vertices in $V\left(C_{n}\right)-W$. If $3 \leq i \leq \frac{n+1}{2}, r\left(v_{i} \mid W\right)=(i-1, i-2)$. If $i=\frac{n+3}{2}, r\left(v_{i} \mid W\right)=\left(\frac{n-1}{2}, \frac{n-1}{2}\right)$. If $\frac{n+5}{2} \leq i \leq n$, $r\left(v_{i} \mid W\right)^{2}=(n-i+1, n-i+2)$. Thus, $W$ is a local resolving set of $C_{n}$. Since $\langle W\rangle$ is connected, it follows that $W$ is a connected local resolving set of $C_{n}$ and so $\operatorname{cld}\left(C_{n}\right) \leq 2$. Since $C_{n}$ is not bipartite, it follows by Theorem 2.1 (i) that cld $\left(C_{n}\right) \geq 2$. Hence, $\operatorname{cld}\left(C_{n}\right)=2$.

Observe that if $G^{\prime}$ is a graph obtained by adding a pendant edge to a connected graph $G$, then it is easy to verify that $\operatorname{cld}\left(G^{\prime}\right)=\operatorname{cld}(G)$. However, if a vertex $v$ is added to a connected graph $G$ such that more than one edge is incident with $v$, then the connected local dimension of the resulting graph can stay the same, decrease, or increase significantly. For example, for $n \geq 3,1 \leq \operatorname{cld}\left(C_{n}\right) \leq 2$. Consider the connected local dimension of a wheel $W_{n}=C_{n}+K_{1}$, where $n \geq 3$. Clearly, $\operatorname{cld}\left(W_{3}\right)=3, \operatorname{cld}\left(W_{4}\right)=\operatorname{cld}\left(W_{5}\right)=$ 2 and $\operatorname{cld}\left(W_{6}\right)=3$. However, for $n \geq 7$, the connected local dimension of a wheel $W_{n}$ increases with $n$ as we show next.

In $W_{n}=C_{n}+K_{1}$, let $C_{n}=\left(v_{1}, v_{2}, \ldots, v_{n}, v_{1}\right)$, where $n \geq 7$, and let $v$ be the central vertex of $W_{n}$. Let $S$ be a set of two or more vertices of $C_{n}$, let $v_{i}$ and $v_{j}$ be two distinct vertices of $S$, and let $P$ and $P^{\prime}$ denote the two distinct $v_{i}-v_{j}$ paths determined by $C_{n}$. If either $P$ or $P^{\prime}$, say $P$, contains only two vertices of $S$ (namely, $v_{i}$ and $v_{j}$ ), then we refer to $v_{i}$ and $v_{j}$ as neighboring vertices of $S$ and the set of vertices of $P$ that belong to $C_{n}-\left\{v_{i}, v_{j}\right\}$ as the gap of $S$ (determined by $v_{i}$ and $v_{j}$ ). The two gaps of $S$ determined by a vertex of $S$ and its two neighboring vertices will be referred to as neighboring gaps. Consequently, if $|S|=r$, then $S$ has $r$ gaps, some of which may be empty.

The next theorem presents a necessary and sufficient condition for a set $W$ to be a local resolving set of $W_{n}$.

Theorem 2.3. Let $W$ be a set of vertices of a wheel $W_{n}=C_{n}+K_{1}$, where $n \geq 7$. Then $W$ is a local resolving set of $W_{n}$ if and only if every gap of $W$ contains at most three vertices of $C_{n}$.

Proof. Assume, to the contrary, that there is a gap of $W$ containing at least four vertices of $C_{n}$. Then there 
are two adjacent vertices $u$ and $u^{\prime}$ in this gap such that $d(u, w)=d\left(u^{\prime}, w\right)=2$ for all $w \in W-\{v\}$. Therefore, $r(u \mid W)=r\left(u^{\prime} \mid W\right)$, which is impossible. To show the converse, suppose that every gap of $W$ contains at most three vertices of $C_{n}$. Since $n \geq 7$, it follows that $W$ contains at least two vertices of $C_{n}$. For any two adjacent vertices $x$ and $y$ contained in a gap of $W$, there exists a vertex in $W$ adjacent to only one of $\{x, y\}$. Hence the representation of $x$ and $y$ with respect to $W$ are distinct. If the central vertex $v \in W$, we are done. Suppose that $v \notin W$.

Case 1. $|W| \geq 3$.

Since $v$ is adjacent to every vertex of $C_{n}$, it follows that the representations of $v$ and any vertices of $C_{n}$ with respect to $W$ are distinct.

Case 2. $|W|=2$.

Suppose that there is a vertex $w^{\prime} \in V\left(C_{n}\right)$ such that $r\left(w^{\prime} \mid W\right)=(1,1)=r(v \mid W)$. Since $n \geq 7, W$ contains a gap of at least four vertices, which is impossible. Hence the representations of $v$ and any vertices of $C_{n}$ with respect to $W$ are distinct.

An immediate consequence from Theorem 2.3 is that if $W$ is a local resolving of $W_{n}$, where $n \geq 7$, $W-\{v\}$ is also a local resolving set. It follows that, for $n \geq 7$, every local basis of $W_{n}$ contains no central vertex. However, every connected local basis of $W_{n}$ must contain the central vertex. It is shown in the next result.

Lemma 2.4. Every connected local basis of a wheel $W_{n}$, where $n \geq 7$ must contain the central vertex.

Proof. Assume, to the contrary, that there is a connected local basis $W$ of $W_{n}$ not containing the central vertex $v$. Then $W$ consists of consecutive vertices in $C_{n}$. Without loss of generality, let $W=$ $\left\{v_{1}, v_{2}, \ldots, v_{k}\right\}$. By Theorem 2.3, it implies that $k \geq n-3$. By the argument similar to the one used for the proof of Theorem 2.3, the set $W^{\prime}=$ $\left\{v, v_{1}, v_{4}, v_{5}, \ldots, v_{k}\right\}$ is a local resolving set of $W_{n}$. Moreover, $\left\langle W^{\prime}\right\rangle$ is connected, that is, $W^{\prime}$ is also a connected local resolving set of $W_{n}$ having cardinality $k-1$, contradicting the assumption that $W$ is a connected local basis of $W_{n}$.

We are now prepared to present the connected local dimension of a wheel $W_{n}$, where $n \geq 7$.

Theorem 2.5. Let $W_{n}$ be a wheel, where $n \geq 7$, Then $\operatorname{cld}\left(W_{n}\right)=\left\lceil\frac{n}{4}\right\rceil+1$.

Proof. By Theorem 2.3 and Lemma 2.4, we obtain that $\operatorname{cld}\left(W_{n}\right) \geq\left\lceil\frac{n}{4}\right\rceil+1$. It remains to verify that $\operatorname{cld}\left(W_{n}\right) \leq\left\lceil\frac{n}{4}\right\rceil+1$. Let $W=\left\{v_{i} \in V\left(C_{n}\right) \mid i \equiv\right.$ $1(\bmod 4)\} \cup\{v\}$ with $|W|=\left\lceil\frac{n}{4}\right\rceil+1$. Since every gap of $W$ contains at most three vertices from $C_{n}$, it follows by Theorem 2.3 that $W$ is a local resolving set of $W_{n}$. Moreover, since $W$ contains the central vertex $v$, it follows that $\langle W\rangle$ is connected and so $W$ is a connected local resolving set of $W_{n}$. Therefore, $\operatorname{cld}\left(W_{n}\right) \leq\left\lceil\frac{n}{4}\right\rceil+1$. Hence, $\operatorname{cld}\left(W_{n}\right)=\left\lceil\frac{n}{4}\right\rceil+1$.

\section{Graphs with prescribed connected local dimensions and other parameters}

The open neighborhood or the neighborhood of a vertex $u$ of a connected graph $G$ is the set of all vertices that are adjacent to $u$, which is denoted by $N(u)=\{v \in V(G) \mid u v \in E(G)\}$. The closed neighborhood $N[u]$ of $u$ is defined as $N(u) \cup\{u\}$. Two vertices $u$ and $v$ of $G$ are twins if either (i) $u v \notin E(G)$ and $N(u)=N(v)$ or (ii) $u v \in E(G)$ and $N[u]=N[v]$. In particular, if the condition (ii) holds, then $u$ and $v$ are called true twins. Consequently, the relations twin and true twin are equivalence relations on $V(G)$ and, as such, these relations partition $V(G)$ into equivalence classes which are called twin equivalence classes and true twin equivalence classes, respectively or, more simply, twin classes and true twin classes, respectively. Observe that if $G$ contains $l$ distinct true twin classes $U_{1}, U_{2}, \ldots, U_{l}$ of $G$, then every local resolving set of $G$ must contain at least $\left|U_{i}\right|-1$ vertices from each $U_{i}$, where $1 \leq i \leq l$. This observation has been described in [2] as we state next.

Proposition 3.1 ([2]). Let $G$ be a connected graph having $l$ true twin classes $U_{1}, U_{2}, \ldots, U_{l}$. Then every local resolving set of $G$ must contain $\left|U_{i}\right|-1$ vertices from each $U_{i}$, where $1 \leq i \leq l$. Moreover, $\operatorname{ld}(G) \geq$ $\sum_{i=1}^{l}\left|U_{i}\right|-l$.

We have seen that if $G$ is a connected graph of order $n$ with $\operatorname{ld}(G)=a$ and $\operatorname{cld}(G)=b$, then $1 \leq a \leq b \leq n-1$ by Eq.(1.1). In fact, any integers $a, b$ and $n$ with $1 \leq a \leq b \leq n-1$ are realizable as the local dimension, connected local dimension and order of some graphs as we show next.

Theorem 3.2. Let $a, b, n$ be integers with $n \geq 4$. Then there exists a connected graph $G$ of order $n$ with $\operatorname{ld}(G)=a$ and $\operatorname{cld}(G)=b$ if and only if $a, b, n$ satisfy one of the following:

(i) $a=b=n-1$,

(ii) $a=b=1$, and

(iii) $2 \leq a \leq b \leq n-2$.

Proof. Assume that there exists a connected graph $G$ of order $n$ with $\operatorname{ld}(G)=a$ and $\operatorname{cld}(G)=b$. By Eq.(1.1), we obtain that $1 \leq a \leq b \leq n-1$. If $b=n-1$, then $G$ is a complete graph $K_{n}$. Thus, 
$a=b=n-1$. If $a=1$, then $G$ is a bipartite graph. Therefore, $a=b=1$. For otherwise, $2 \leq a \leq b \leq c \leq n-2$. Hence, if $G$ is a connected graph of order $n$ with $\operatorname{ld}(G)=a$ and $\operatorname{cld}(G)=b$, then $a, b$ and $n$ must satisfy one of (i), (ii) and (iii). It remains to verify the converse. If $a=b=n-1$, then let $G$ be a complete graph $K_{n}$ and the result is true. If $a=b=1$, then let $G$ be a path $P_{n}$. Thus, the graph $G$ has the desired properties. We may assume that $2 \leq a \leq b \leq n-2$. We consider two cases.

Case 1. $a=b$.

Let $G^{\prime}$ be a graph obtained from a complete graph $K_{a}$ with vertex set $\left\{u_{1}, u_{2}, \ldots, u_{a}\right\}$ and a path $P_{n-a}=$ $\left(v_{1}, v_{2}, \ldots, v_{n-a}\right)$ by joining $v_{1}$ to every vertex of $K_{a}$. Since $V\left(K_{a}\right)$ is a true twin class of $G^{\prime}$, every local resolving set of $G^{\prime}$ must contain at least $a-1$ vertices from $V\left(K_{a}\right)$. However, if a set $W$ contains only $a-1$ vertices from $V\left(K_{a}\right)$, then $W$ does not contain $u_{i}$ for some integer $i$ with $1 \leq i \leq a$ and so $r\left(u_{i} \mid W\right)=r\left(v_{1} \mid W\right)=(1,1, \ldots, 1)$. Therefore, $G$ contains no local resolving set of cardinality $a-1$, that is, $\operatorname{ld}\left(G^{\prime}\right) \geq a$. Since $r\left(v_{j} \mid V\left(K_{a}\right)\right)=(j, j, \ldots, j)$, where $1 \leq j \leq n-a$, it follows that $V\left(K_{a}\right)$ is a local resolving set of $G^{\prime}$ having cardinality $a$, that is, $V\left(K_{a}\right)$ is a local basis of $G^{\prime}$. Moreover, $V\left(K_{a}\right)$ is also a connected local basis of $G^{\prime}$. Hence, $\operatorname{ld}\left(G^{\prime}\right)=$ $\operatorname{cld}\left(G^{\prime}\right)=a$.

Case 2. $a<b$.

Let $G$ be a graph obtained from a complete graph $K_{a}$ with vertex set $\left\{u_{1}, u_{2}, \ldots, u_{a}\right\}$ and two paths $P_{b-a+1}=\left(v_{1}, v_{2}, \ldots, v_{b-a+1}\right)$ and $P_{n-b-1}=$ $\left(w_{1}, w_{2}, \ldots, w_{n-b-1}\right)$ by joining $v_{1}$ to every vertex of $K_{a}$, and $w_{1}$ to both $v_{b-a}$ and $v_{b-a+1}$. Since $V\left(K_{a}\right)$ is a true twin class of $G$, it follows by Proposition 3.1 that every local resolving set of $G$ must contain at least $a-1$ vertices from $V\left(K_{a}\right)$. However, every set consisting of $a-1$ vertices from $V\left(K_{a}\right)$ is not a local resolving set of $G$ since the representations of $v_{b-a+1}$ and $w_{1}$ with respect to this set are the same. Thus, every local resolving set of $G$ contains at least $a$ vertices. It is routine to verify that every local resolving set of $G$ must contain at least one vertex from $\left\{v_{b-a+1}\right\} \cup V\left(P_{n-b-1}\right)$. Then the set $\left(V\left(K_{a}\right)-\left\{u_{1}\right\}\right) \cup\left\{v_{b-a+1}\right\}$ is a minimum local resolving set of $G$. Hence, $\operatorname{ld}(G)=a$. Since every connected local resolving set of $G$ is also a local resolving set of $G$, it follows that every connected local resolving set of $G$ must contain at least $a-1$ vertices from $V\left(K_{a}\right)$ and at least one vertex from $\left\{v_{b-a+1}\right\} \cup V\left(P_{n-b-1}\right)$. Therefore, every connected local resolving set of $G$ contains $v_{1}, v_{2}, \ldots, v_{b-a}$. In fact, the set $\left(V\left(K_{a}\right)-\left\{u_{1}\right\}\right) \cup V\left(P_{b-a+1}\right)$ is a connected local basis of $G$, that is, $\operatorname{cld}(G)=b$.

We know by Eq.(1.2) that if $G$ is a connected graph of order $n$ with $\operatorname{cld}(G)=b$ and $\operatorname{cd}(G)=c$, then $1 \leq$ $b \leq c \leq n-1$. Next, we show that for any integers $b, c$ and $n$ with $1 \leq b \leq c \leq n-1$ are realizable as the connected local dimension, connected dimension and order of some graphs.

Theorem 3.3. Let $b, c, n$ be integers with $n \geq 4$. Then there exists a connected graph $G$ of order $n$ with $\operatorname{cld}(G)=b$ and $\operatorname{cd}(G)=c$ if and only if $b, c, n$ satisfy one of the following:

(i) $b=c=n-1$,

(ii) $b=1$ and $1 \leq c \leq n-1$, and

(iii) $2 \leq b \leq c \leq n-2$.

Proof. Assume that there exists a connected graph of order $n$ with $\operatorname{cld}(G)=b$ and $\operatorname{cd}(G)=c$. By Eq.(1.2), we obtain that $1 \leq b \leq c \leq n-1$. If $b=n-1$, then $c=n-1$ by Eq.(1.2). If $b=1$, then $1 \leq c \leq n-1$ by Eq.(1.2). If $2 \leq b \leq n-2$, then $G$ is neither a star nor a complete graph, and so $2 \leq b \leq c \leq n-2$. Hence, if $G$ is a connected graph of order $n$ with $\operatorname{cld}(G)=b$ and $\operatorname{cd}(G)=c$, then $b, c$ and $n$ must satisfy one of (i), (ii) and (iii). It remains to verify the converse. If $b=c=n-1$, then let $G$ be a complete graph $K_{n}$ and the result is true. Next, assume that $b=1$ and $1 \leq c \leq n-1$. For $c=1$, let $G$ be a path $P_{n}$; while for $c=n-1$ let $G$ be a star $K_{1, n-1}$. Since $\operatorname{cld}\left(P_{n}\right)=\operatorname{cd}\left(P_{n}\right)=1$, and $\operatorname{cld}\left(K_{1, n-1}\right)=1$ and $\operatorname{cd}\left(K_{1, n-1}\right)=n-1$, it follows that the result holds for $b=1$ and $c=1, n-1$. For $2 \leq c \leq n-2$, let $G$ be a graph obtained from a complete bipartite graph $K_{2, c-1}$ with partite sets $U=\left\{u_{1}, u_{2}\right\}$ and $U^{\prime}=\left\{w_{1}, w_{2}, \ldots, w_{c-1}\right\}$, and a path $P_{n-c-1}=\left(v_{1}, v_{2}, \ldots, v_{n-c-1}\right)$ by joining $v_{1}$ to both $u_{1}$ and $u_{2}$. Since $G$ is bipartite, it follows that $\operatorname{cld}(G)=1$. It is routine to show that the set $V\left(K_{2, c-1}\right)-\left\{u_{2}\right\}$ is a connected basis of $G$. Therefore, $\operatorname{cd}(G)=c$. Hence, the result holds for $b=1$ and $2 \leq c \leq n-2$. Now assume that $2 \leq b \leq c \leq n-2$. We consider two cases.

Case 1. $b=c$.

The graph $G^{\prime}$ of the proof for Theorem 3.2 has $\operatorname{cld}\left(G^{\prime}\right)=b$ with a connected local basis $V\left(K_{b}\right)$. In fact, $V\left(K_{b}\right)$ is also a connected basis of $G^{\prime}$, that is, $\operatorname{cd}\left(G^{\prime}\right)=b$.

Case 2. $b<c$.

Let $G$ be a graph obtained from a complete graph $K_{b}$ with vertex set $\left\{u_{1}, u_{2}, \ldots, u_{b}\right\}$, a star $K_{1, c-b}$ with vertex set $\left\{v, v_{1}, v_{2}, \ldots, v_{c-b}\right\}$ and a path $P_{n-c-1}=$ $\left(w_{1}, w_{2}, \ldots, w_{n-c-1}\right)$ by joining the central vertex $v$ of $K_{1, c-b}$ to $w_{1}$ and every vertex of $K_{b}$. It is immediate that the set $V\left(K_{b}\right)$ is a connected local basis of $G$. Therefore, $\operatorname{cld}(G)=b$. Moreover, the set $\left(V\left(K_{b}\right)-\left\{u_{1}\right\}\right) \cup V\left(K_{1, c-b}\right)$ is a connected basis of $G$, that is, $\operatorname{cd}(G)=c$. 


\section{Connected local bases and local bases in graphs}

In this section, we study the relationship between connected local bases and local bases in a connected graph $G$. Certainly, if $W$ is a local resolving set of $G$, then a set $W^{\prime}$ containing $W$ is also a local resolving set of $G$. Therefore, if $W$ is a local basis of $G$ such that $\langle W\rangle$ is disconnected, then surely there is a smallest superset $W^{\prime}$ of $W$ for which $\left\langle W^{\prime}\right\rangle$ is connected. This suggests the following question: Does there exist a graph with a connected local basis not containing any local bases? The answer to this question is given in the next result.

Theorem 4.1. There is an infinite class of connected graphs $G$ such that some connected local bases of $G$ contain a local basis of $G$ and others contain no local basis of $G$.

Proof. Let $G$ be a graph obtained from a complete graph $K_{a}$ of order $a \geq 2$ with vertex set $\left\{u_{1}, u_{2}, \ldots, u_{a}\right\}$, a cycle $C_{4}=\left(v_{1}, v_{2}, v_{3}, v_{4}, v_{1}\right)$ and a path $P_{3}=\left(w_{1}, w_{2}, w_{3}\right)$ by joining $v_{1}$ to every vertex of $K_{a}$ and joining $w_{1}$ and $w_{3}$ to $v_{1}, v_{4}$ and $v_{2}, v_{3}$, respectively. A graph $G$ is shown in Fig. 2. We first

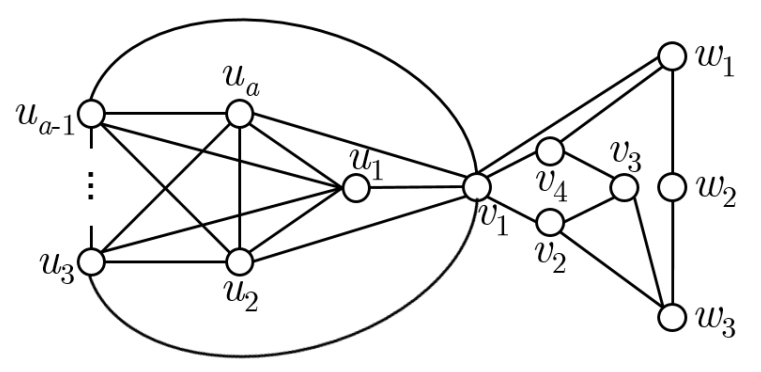

Figure 2: A graph $G$

verify that the set $B=\left\{u_{1}, u_{2}, \ldots, u_{a-1}\right\} \cup\left\{w_{2}\right\}$ is a local basis of $G$. We can show, by a case-by-case analysis, that $B$ is a local resolving set of $G$. Next, we claim that $B$ is a local resolving set of minimum cardinality. Assume, to the contrary, that there is a local resolving set $W$ of $G$ having cardinality at most $a-1$. Since $V\left(K_{a}\right)$ is a true twin class of $G$, it follows that every local resolving set of $G$ must contain at least $a-1$ vertices of $K_{a}$. Therefore, $W$ consists of $a-1$ vertices of $K_{a}$. However, $v_{4}$ and $w_{1}$ are adjacent and $d\left(v_{4}, u_{i}\right)=d\left(w_{1}, u_{i}\right)$ for each integer $i$ with $1 \leq i \leq a$. This is a contradiction. Hence, $B$ is a local basis of $G$ and so $\operatorname{ld}(G)=a$. Second, we determine that $\operatorname{cld}(G)=a+2$. In order to do this, we claim that $\operatorname{cld}(G) \geq a+2$. Suppose, contrary to our claim, that there is a connected local resolving set $W^{\prime}$ of $G$ having cardinality $a+1$. Recall that every connected local basis of $G$ must contain at least $a-1$ vertices of $K_{a}$. We consider two cases.

Case 1. $V\left(K_{a}\right) \subseteq W^{\prime}$.

Since $\left\langle W^{\prime}\right\rangle$ is connected and $\left|W^{\prime}\right|=a+1$, it follows that $W^{\prime}=V\left(K_{a}\right) \cup\left\{v_{1}\right\}$. However, since $v_{4}$ is adjacent to $w_{1}$ and $r\left(v_{4} \mid W^{\prime}\right)=r\left(w_{1} \mid W^{\prime}\right)$, it follows that $W^{\prime}$ is not a connected resolving set of $G$, which is a contradiction.

Case 2. $V\left(K_{a}\right) \nsubseteq W^{\prime}$.

Since $\left\langle W^{\prime}\right\rangle$ is connected and $\left|W^{\prime}\right|=a+1$, it follows that $W^{\prime}$ contains $v_{1}$ and one vertex from $\left\{v_{2}, v_{4}, w_{1}\right\}$. If $W^{\prime}$ contains $v_{2}$ or $w_{1}$, then $r\left(v_{3} \mid W^{\prime}\right)=r\left(w_{3} \mid W^{\prime}\right)$. If $W^{\prime}$ contains $v_{4}$, then $r\left(w_{2} \mid W^{\prime}\right)=r\left(w_{3} \mid W^{\prime}\right)$. Therefore, $W^{\prime}$ is not a connected local resolving set of $G$. This is also a contradiction.

Therefore, $\operatorname{cld}(G) \geq a+2$. On the other hand, the sets $S_{1}=\left\{u_{1}, u_{2}, \ldots, u_{a-1}\right\} \cup\left\{v_{1}, w_{1}, w_{2}\right\}$ and $S_{2}=\left\{u_{1}, u_{2}, \ldots, u_{a-1}\right\} \cup\left\{v_{1}, v_{4}, w_{1}\right\}$ are connected local resolving sets of $G$. Therefore, $\operatorname{cld}(G) \leq a+2$. Hence, $\operatorname{cld}(G)=a+2$.

Last, it can be verified that every local basis of $G$ contains exactly $a-1$ vertices of $K_{a}$ and exactly one vertex from $\left\{v_{3}, w_{2}\right\}$. Observe that the connected local basis $S_{1}$ contains the local basis $B$ of $G$, while the connected local basis $S_{2}$ contains no local basis of $G$.

From the previous theorem, there is a connected graph having many connected local bases. This leads us to determine a connected graph $G$ having a unique connected local basis. It has been shown in [2] that there is a connected graph with a unique local basis. In fact, there is a connected graph with a unique connected local basis as we show next.

Theorem 4.2. For $k \geq 3$, there exists a graph with a unique connected local basis of cardinality $k+1$.

Proof. Let $G_{1}$ be a complete graph $K_{2^{k}}$ with vertex set $U=\left\{u_{0}, u_{1}, \ldots, u_{2^{k}-1}\right\}$, and let $G_{2}$ be a empty graph $\bar{K}_{k}$ with vertex set $W=\left\{w_{k-1}, w_{k-2}, \ldots, w_{0}\right\}$. Then the graph $G$ is obtained from $G_{1}$ and $G_{2}$ by adding edges between $U$ and $W$ as follows. Let each integer $j$ for $1 \leq j \leq 2^{k}-1$ be expressed in its base 2 (binary) representation. Thus, each such $j$ can be expressed as a sequence of $k$ coordinates, that is, a $k$ vector, where the rightmost coordinate represents the value (either 0 or 1 ) in the $2^{0}$ position, the coordinate to its immediate left is the value in the $2^{1}$ position, etc. For integers $i$ and $j$ with $0 \leq i \leq k-1$ and $0 \leq j \leq 2^{k}-1$, we join $w_{i}$ and $u_{j}$ if and only if the value in the $2^{i}$ position in the binary representation of $j$ is 1 . For example, Fig. 3 shows the edges joining between $U$ and $W$ in the graph $G$ for $k=3$. It was shown in [2] that $W$ is a unique local basis of $G$. Therefore, there is no connected local basis of $G$ having cardinality $k$, that is, $\operatorname{cld}(G) \geq k+1$. Since $W$ is a local basis of $G$, it follows that $W^{\prime}=W \cup\left\{u_{2^{k-1}}\right\}$ 


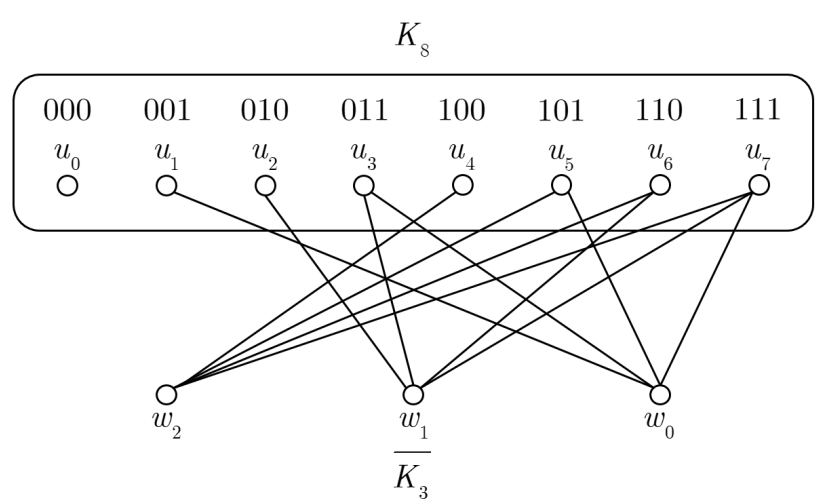

Figure 3: A graph $G$ for $k=3$

is a connected local resolving set of $G$. In fact, $W^{\prime}$ is a connected local basis of $G$.

It remains only to show that $G$ has no other connected local basis. If $U^{\prime} \subseteq U$ and $\left|U^{\prime}\right|=k+1$, then $\left|U-U^{\prime}\right|=2^{k}-k-1 \geq 2$. Since the distance of every two vertices of $U$ is 1 , it follows that there are at least two adjacent vertices of $U-U^{\prime}$ having the same representation with respect to $U^{\prime}$, and so $U^{\prime}$ is not a connected local resolving set of $G$. Thus, every connected local resolving set of $G$ must contain at least one vertex of $W$. Suppose that $B \neq W^{\prime}$ is a connected local basis of $G$. Therefore, $B=U^{\prime \prime} \cup W^{\prime \prime}$, where $U^{\prime \prime} \subseteq U$ and $W^{\prime \prime} \subseteq W$. If $\left|W^{\prime \prime}\right|=k$, then $B$ does not contain $u_{2^{k}-1}$. Therefore, $\langle B\rangle$ is not connected, which is impossible. If $\left|W^{\prime \prime}\right| \leq k-1$, then $U^{\prime \prime}$ contains at least two vertices. We may therefore assume that $\left|U^{\prime \prime}\right|=i \geq 2$. Then $\left|W^{\prime \prime}\right|=k-i+1$. Since every vertex of $U-U^{\prime \prime}$ has distance 1 from every vertex of $U^{\prime \prime}$, it follows that there are at most $2^{k-i+1}$ distinct representations of vertices of $U-U^{\prime \prime}$ with respect to $B$. However, since $2^{k}-i>2^{k-i+1}$, there are two vertices of $U-U^{\prime \prime}$ such that their representation with respect to $B$ are the same, contradicting the fact that $B$ is a connected local basis of $G$. Hence, $W^{\prime}$ is a unique connected local basis of $G$.

\section{Discussion and conclusions}

By Eq.(1.3), it suggests the following question: For which quadruples $a, b, c, n$ of integers with $1 \leq a \leq$ $b \leq c \leq n-1$, does there exist a connected graph $G$ of order $n$ with $\operatorname{ld}(G)=a, \operatorname{cld}(G)=b$ and $\operatorname{cd}(G)=c$ ?

In this research, we have investigated the connected local dimensions of bipartite graphs, complete graphs, cycles and wheels. We show the realization results that any integers $a, b$ and $n$ with $1 \leq$ $a \leq b \leq n-1$ are realizable as the local dimension, connected local dimension and order of some graphs. Moreover, for any integers $b, c$ and $n$ with $1 \leq b \leq c \leq n-1$ are realizable as the connected local dimension, connected dimension and order of some graphs. We present the relationship between connected local bases and local bases in a connected graph, that is, there is an infinite class of connected graphs $G$ such that some connected local bases of $G$ contain a local basis of $G$ and others contain no local basis of $G$. We determine the stronger result that there is a connected graph with a unique connected local basis.

\section{References:}

[1] G. Chartrand, L. Eroh, M. Johnson \& O.R Oellermann. (2000). Resolvability in graphs and the metric dimension of a graph. Discrete Applied Mathematics, 105, 99-113.

[2] F. Okamoto, B. Phinezy \& P. Zhang. (2010). The local metric dimension of a graph. Mathematical Bohemica, 135(3), 239-255.

[3] F. Harary \& R. A. Melter. (1976). On the metric dimension of a graph. Ars Combinatoria, 2, 191-195.

[4] B. L. Hulme, A. W. Shiver \& P. J. Slater. (1981). FIRE: a subroutine for fire protection network analysis (SAND 81-1261). New Mexico: Sandia National Laboratories.

[5] B. L. Hulme, A. W. Shiver \& P. J. Slater. (1982) Computing minimum cost fire protection (SAND 82-0809). New Mexico: Sandia National Laboratories.

[6] B. L. Hulme, A. W. Shiver \& P. J. Slater. (1984). A Boolean algebraic analysis of fire protection. North-Holland Mathematics Studies, 95, 215227.

[7] S. Isariyapalakul, V. Khemmani \& S. W. Pho-on. (2020). The multibases of symmetric caterpillars. Journal of Mathematics, 2020 doi: $10.1155 / 2020 / 5210628$

[8] M. A. Johnson. (1998). Browsable structureactivity datasets. Advances in Molecular Similarity, 2, 153-170.

[9] V. Khemmani \& S. Isariyapalakul. (2018). Multiresolving sets of graphs with prescribed multisimilar equivalence classes. International Journal of Mathematics and Mathematical Sciences, 2018 doi: $10.1155 / 2018 / 8978193$

[10] V. Khemmani \& S. Isariyapalakul. (2020). The characterization of caterpillars with multidimension 3. Thai Journal of Mathematics, 2020(Special Issue), 247-259. 
[11] S. Khuller, B. Rsghavachari \& A. Rosenfeld. (1994). Localization in graphs (CS-TR-3326). Maryland: University of Maryland.

[12] V. Saenpholphat \& P. Zhang. (2003). Connected resolvability of graphs. Czechoslovak Mathematical Journal, 53, 827-840.

[13] P. J. Slater. (1975). Leaves of trees. Congressus Numerantium, 14, 549-559.

[14] P. J. Slater. (1998). Dominating and reference sets in graphs. Journal of Mathematical Physics, Sci, 22, 445-455.

[15] J. Wang, L. Miao \& Y. Liu. (2019) Characterization of $n$-vertex graphs of metric dimension $n-3$ by metric matrix. Mathematics, 7(479), $1-13$.

\section{Sources of funding for research presented in a scientific article or scientific article itself}

This research was supported by Faculty of Science, Srinakharinwirot University.

\section{Creative Commons Attribution \\ License 4.0 (Attribution 4.0 \\ International, CC BY 4.0)}

This article is published under the terms of the Creative Commons Attribution License 4.0

https://creativecommons.org/licenses/by/4.0/deed.en US 